\title{
The pictorial superiority effect in recognition memory
}

\author{
JOAN GAY SNODGRASS and ANTHONY ASIAGHI \\ New York University, New York, New York 1000 s
}

\begin{abstract}
To test the hypothesis, recently advanced by Nelson, Reed, and Walling (1976), that pictures are more memorable than words because of a superior sensory rather than conceptual code, concept memory was tested so that stimulus form could be divorced from stimulus meaning. In a recognition memory task, subjects in two experimental conditions were instructed to identify test items as "old" only if they appeared in the opposite form from their study form (i.e., study pictures tested as words and study words tested as pictures), and to identify as "new" previously shown items tested in the same form and completely new items. Memory of old concepts was identical for experimental groups compared to control groups, who were tested with same-form items, and there was no difference between groups instructed prior to study about the changed-form tests and those instructed only prior to test. Thus, changing the rule for identifying old and new items had no effect on recognition memory, nor did providing an opportunity for differential encoding by prior instructions. The present results suggest that concepts represented as pictures are remembered better than concepts represented as words, regardless of how such memory is tested, and thus that the pictorial superiority effect stems from superior meaning codes as well as superior sensory codes.
\end{abstract}

Recently, Nelson, Reed, and Walling (1976) investigated the locus of the pictorial superiority effect. In a paired associate recall task, they varied both conceptual and sensory similarity among pictures and words, and found that the pictorial superiority effect occurred only when pictures were low in sensory similarity. An increase in visual similarity eliminated or even reversed the pictorial superiority effect, whereas conceptual similarity had equivalent effects on both pictures and words. Hence, they concluded that pictures are remembered better because of their more elaborate sensory codes.

Pictures have consistently been shown to be superior to words in recognition memory studies (e.g., Paivio, 1969; Snodgrass \& McClure, 1975). However, in the typical recognition memory experiment, memory for studied items is assessed by testing items in the same form as studied interspersed with lures of the same form. If more distinctive sensory codes underlie the picture superiority effect, then subjects could show superior recognition of both old pictures and new pictures because of their more distinctive sensory codes.

To test the sensory code hypothesis, it is necessary to divorce the sensory aspects of the concept as represented from the concept's meaning. One way of doing this is to test old pictures by their verbal labels. Although some investigators have found a slight decrement in memory for pictures tested with their name compared to pictures tested with the identical pictures (Jenkins, Neale, \& Deno, 1967), others have not (Paivio, in press; Snodgrass $\&$ McClure, 1975), and in any case the decrement observed from changed-form tests is not large enough to reduce picture performance to the level of word performance.
Alternatively, picture superiority in recognition may be partly attributable to the ability of subjects to recognize new pictures as new better than new words. To test this possibility, we need a procedure for divorcing the old/new decision from test item form. In the present experiment, that was accomplished by instructing an experimental group of subjects to consider as old only those items tested in a form opposite from the study form, in contrast to a control group, which was instructed to consider as old only those items tested in the same form as the study form.

An additional purpose of the present experiment was to evaluate the effect of prior instructions on performance when the old/new criterion was changed. It was hypothesized that subjects who were forewarned about the changed criterion for old/new decisions would be more likely to encode both the concept itself and its form class during study, and hence perform at a higher level of accuracy than uninstructed subjects.

\section{METHOD}

\section{Subjects}

Seventy-two New York University undergraduates, of whom 37 were female and 35 were male, participated in the experiment to fulfill a course requirement. They were randomly divided into four groups of 18 subjects each and were assigned to one of the four conditions: control uninstructed, control instructed. experimental uninstructed, and experimental instructed.

\section{Apparatus and Materials}

A total of 160 slides was used in the experiment. Half of the slides were line drawings of common objects and the other half were their corresponding names. The line drawings were obtained by having a pilot group of 35 subjects draw the simplest picture they could think of for each of 100 concepts, and 
Table 1

Classification of Test Items and Their Notation

\begin{tabular}{clllll}
\hline & \multicolumn{4}{c}{ Test Form } \\
\cline { 2 - 3 } Study Form & \multicolumn{2}{c}{ Singly Tested } & & \multicolumn{2}{c}{ Doubly Tested } \\
\cline { 2 - 3 } \cline { 5 - 6 } & P & W & & P & W \\
\hline \multirow{2}{*}{ P } & PP & PW & & PP(W) & P(P)W \\
& $(10)$ & $(10)$ & & $(10)$ & $(10)$ \\
W & WP & WW & & WP(W) & W(P)W \\
& $(10)$ & $(10)$ & & $(10)$ & $(10)$ \\
& $-P$ & $-W$ & & \\
\hline
\end{tabular}

Vote- $-P$ and $-W$ are completely new items. The numbers in parentheses are the number of different items in each category.

then choosing representative examples of each. The 80 slides actually used were selected by showing another pilot group of 18 subjects the 100 slides via a slide projector and asking them to label each drawing. Of the 100,80 were given identical labels by at least 15 of the 18 subjects, and these stimuli were used in the experiment in both their picture and word forms.

\section{Procedure}

The experiment consisted of two phases. In the study phase, 60 stimulus items were presented. Half of these were pictures and half were unrelated words. In the test phase, 100 items were presented in randomized order, divided as follows: (a) 20 pictures and 20 words previously presented in the study phase in the same form; (b) 20 pictures and 20 words previously presented in the study phase in the opposite form (i.e., the pictures were originally presented as words, and the words were originally presented as pictures); (c) 10 pictures and 10 words which were completely new. In addition, half of the old items were tested in both their picture and word forms, so that 10 pictures in Category a also appeared as 10 words in Category b, and 10 words in Category a also appeared as 10 pictures in Category b.

This resulted in eight categories of test items, defined by whether the items were old or new, pictures or words, and, for old items, by whether they were in the same or different form as the original items and whether the items were singly or doubly tested. Table 1 summarizes the distribution of items and their notation. Although the doubly tested items comprise only one category of concepts, they can be divided into two categories according to the form of their test, so the total number of test items is 100 . Words across the eight categories were matched for frequency according to the Kučera and Francis (1967) norms, and the average frequencies ranged from 75 to 115 .
Subjects were randomly assigned to one of four groups, of which two were experimental and two were control. The experimental and control groups differed by the criteria they were to use to classify test items as old or new. The experimental group was instructed to classify as "old" only items they had studied in a different form, and to classify as "new" studied items presented in the same form and completely new items. The control group was instructed to classify as "old" only items they had studied in the same form, and to classify as "new" studied items presented in a different form and completely new items.

The instructions about the criteria of classification were given either before the study phase (instructed group) or after the study phase (uninstructed group). Subjects were tested in groups of 10 to 21 . Instructed and uninstructed groups were tested separately, but experimental and control groups were tested during the same session. Subjects were randomly assigned to the experimental or control condition by which answer sheet they received, and the experimental subjects were told to ignore the instructions given to the control subjects and vice versa.

Each stimulus item was exposed for 8 sec during both study and test. The interval between the study and test phases was occupied with instructions for the uninstructed groups and with signing subject permission forms for the instructed groups; the interval was the same duration for all groups. During the test phase, subjects first identified each item as "old" or "new" and then rated the confidence of their judgments on a 3-point scale.

\section{RESULTS}

Table 2 presents the average hit and correct rejection rates for the control and experimental groups under instructed and uninstructed conditions. The definition of a hit changes between the experimental and control conditions, so that PP and WW items are hits in the control conditions, while PW and WP items are hits in the experimental conditions.

For the doubly tested items, two hit or correct rejection rates are shown. For control conditions, $\mathrm{PP}(\mathrm{W})$ refers to a hit and $\mathrm{P}(\mathrm{P}) \mathrm{W}$ refers to a correct rejection when old pictures are tested in both forms. Similarly, W(P)W refers to a hit and WP(W) refers to a correct rejection when old words are tested in both forms. Conversely, for the experimental conditions, $\mathrm{P}(\mathrm{P}) \mathrm{W}$ refers to a hit and $\mathrm{PP}(\mathrm{W})$ refers to a correct rejection when old pictures are tested in both forms,

Table 2

Mean Hit and Correct Rejection Rates for the Eight Classes of Study-Test Trials for the Four Groups of Subjects

\begin{tabular}{|c|c|c|c|c|c|c|c|c|}
\hline \multirow[b]{3}{*}{ Study Form } & \multicolumn{4}{|c|}{ Control } & \multicolumn{4}{|c|}{ Experimental } \\
\hline & \multicolumn{4}{|c|}{ Test Form } & \multicolumn{4}{|c|}{ Test Form } \\
\hline & $\mathbf{P}$ & $P(W)$ & w & (P)W & $\mathbf{P}$ & $P(W)$ & W & (P)W \\
\hline \multicolumn{9}{|c|}{ Instructed } \\
\hline $\begin{array}{l}\text { P } \\
\text { W } \\
-\end{array}$ & $\begin{array}{l}.833 \\
.955 \\
.961\end{array}$ & $\begin{array}{l}.922 \\
.950\end{array}$ & $\begin{array}{l}.944 \\
.761 \\
.794\end{array}$ & $\begin{array}{l}.900 \\
.706\end{array}$ & $\begin{array}{l}.906 \\
.706 \\
.866\end{array}$ & $\begin{array}{l}.917 \\
.733\end{array}$ & $\begin{array}{l}.844 \\
.938 \\
.983\end{array}$ & $\begin{array}{l}.811 \\
.883\end{array}$ \\
\hline \multicolumn{9}{|c|}{ Uninstructed } \\
\hline $\begin{array}{l}\text { P } \\
\mathbf{W} \\
-\end{array}$ & $\begin{array}{l}.917 \\
.944 \\
.961\end{array}$ & $\begin{array}{l}.888 \\
.961\end{array}$ & $\begin{array}{l}.855 \\
.717 \\
.833\end{array}$ & $\begin{array}{l}.900 \\
.772\end{array}$ & $\begin{array}{l}.961 \\
.772 \\
.822\end{array}$ & $\begin{array}{l}.961 \\
.756\end{array}$ & $\begin{array}{l}.828 \\
.933 \\
.972\end{array}$ & $\begin{array}{l}.867 \\
.894\end{array}$ \\
\hline
\end{tabular}

.Vote $-P(W)$ are doubly tested items tested with pictures and $(P) W$ are doubly tested items tested with words. Hit rates are in italics. 
Table 3

Mean Hit and Correct Rejection Rates Combined Across Singly and Doubly Tested Items and Across Instructional Conditions

\begin{tabular}{ccccccc}
\hline & \multicolumn{2}{c}{ Control } & & \multicolumn{2}{c}{ Experimental } \\
\cline { 2 - 3 } \cline { 5 - 6 } Study & \multicolumn{2}{c}{ Test Form } & & \multicolumn{2}{c}{ Test Form } \\
Form & $\mathrm{P}$ & & $\mathrm{W}$ & & $\mathrm{P}$ & $\mathrm{W}$ \\
\hline $\mathrm{P}$ & .890 & .900 & & .936 & .838 \\
W & .952 & .739 & & .742 & .912 \\
- & .961 & .814 & & .844 & .978 \\
\hline
\end{tabular}

Note-Hit rates are in italics.

and $\mathrm{WP}(\mathrm{W})$ refers to a hit and $\mathrm{W}(\mathrm{P}) \mathrm{W}$ refers to a correct rejection for old words tested in both forms.

In order to reduce the complexity of the data analysis, hit and correct rejection rates were combined across conditions whenever they did not differ significantly. An inspection of Table 2 reveals very similar patterns of results between instructed and uninstructed groups within each condition. The results of $t$ tests between the hit and correct rejection rates of the two instructional conditions for each of the 10 test item groups in each condition revealed no significant differences (all ts $<1.96$, $\mathrm{df}=34$ ). Accordingly, those rates were combined across the two instructional conditions for the control and experimental groups.

Similarly, $t$ tests betweeen singly and doubly tested items of the same study and test form on the hit or correct rejection rates showed no significant differences (all ts $<1.40, \mathrm{df}=35$ ), so the rates for singly and doubly tested items were combined. These averaged rates are shown in Table 3.

Table 3 reveals that the control and experimental groups are quite similar in their hit rates (shown in italics). Picture hits (PP trials for control and PW for experimental) are approximately equivalent and word hits (WW trials for control and WP trials for experimental) are almost exactly equal. Similarly, correct rejections for completely new pictures $(-\mathrm{P})$ in the control group are approximately equal to correct rejections for completely new words $(-W)$ in the experimental group, and $-\mathrm{W}$ in control is approximately equal to $-\mathrm{P}$ in experimental. However, correct rejections for old concepts tested as pictures are higher for both groups (WP in control and PP in experimental) than correct rejections for old concepts tested as words.

A 2 by 2 analysis of variance was performed on the four correct rejection rates for each group of subjects, with one factor form of the test ( $\mathrm{P}$ vs $\mathrm{W})$ and the other factor old concept vs new concept. For the control group, both the picture/word factor and the oldconcept/new-concept factor were significant but the interaction was not $[\mathrm{Fs}(1,35)=18.93$ and 5.17 and MSes $=.019$ and .011 , respectively]. For the experimental group, both the picture/word factor and the interaction were significant, but the old-concept/new- concept factor was not $[\operatorname{Fs}(1,35)=9.05$ and 40.89 , MSes $=.012$ and .005 , respectively] . Thus, while picture tests for the control group act like word tests for the experimental group (and vice versa) for completely new items, picture tests are uniformly better for both groups when old concepts are being tested.

To obtain an overall measure of recognition performance, the nonparametric measure $\mathrm{A}^{\prime}$ was computed for each subject for both picture and word stimuli. This was accomplished for the control conditions by combining picture hits (PP) with completely new picture false alarms $(-\mathrm{P})$ for picture memory, and by combining word hits (WW) with completely new word false alarms $(-\mathrm{W})$ for word memory.

In the experimental conditions, picture hits come from the PW condition and word hits from the WP condition. However, it is not clear which false alarm rate to use. Should we use a false alarm rate based on the form of the study item (i.e., $-\mathrm{P}$ paired with PW) or according to the form of the test item (i.e., $-W$ paired with PW)? Elsewhere, we have argued that when analyzing memory performance for changed-form items, the appropriate pairing is one based on form of test item rather than form of study item (Snodgrass \& McClure, 1975).

Furthermore, $-\mathrm{P}$ and $-\mathrm{W}$ rates for the experimental groups correspond most closely to the opposite form correct rejection rates (-W and $-\mathrm{P})$ for the control group. Thus, while new picture correct rejection rates are typically higher than new word correct rejection rates, we have reversed this relationship in the experimental group by changing the criteria for classifying items as old. For both these reasons, then, we used the form of the test item as the basis for pairing hit and false alarm rates.

Although our previous analysis of hit and correct rejection rates revealed no differences between instructed and uninstructed groups in either the control or experimental conditions, it seemed possible that some effect of instructions might be observed when the two measures are combined in the $\mathrm{A}^{\prime}$ analysis. Accordingly, average $\mathrm{A}^{\prime}$ 's were computed separately for the two instructional groups.

Table 4 presents mean $\mathrm{A}^{\prime}$ values for the four groups of subjects for pictures and words. There appear to be no differences between experimental and control groups in memory performance, and no differences between instructed and uninstructed groups in memory per-

Table 4

Mean A' Values for the Four Groups of Subjects

\begin{tabular}{lcccccc}
\hline & \multicolumn{2}{c}{ Control } & & \multicolumn{2}{c}{ Experimental } \\
\cline { 2 - 3 } \cline { 6 - 7 } & \multicolumn{2}{c}{ Study Form } & & \multicolumn{2}{c}{ Study Form } \\
& $\mathrm{P}$ & $\mathrm{W}$ & & $\mathrm{P}$ & $\mathrm{W}$ \\
\hline Instructed & .972 & .860 & & .963 & .885 \\
Uninstructed & .978 & .889 & & .964 & .881 \\
\hline
\end{tabular}


formance, and no differences between instructed and uninstructed groups in either condition, but large differences between pictures and words. These impressions are supported by the results of a mixed threeway analysis of variance on the arc sine transforms of the $A^{\prime}$ values with one (between subjects) factor experimental vs control, the second (between subjects) factor instructed vs uninstructed, and the third (within subjects) factor pictures vs words. The only significant main effect was pictures vs words $[F(1,72)=134.68, p<.001$, $\mathrm{MSe}=.035]$. All other main effects and all interactions were insignificant.

The results of this analysis of variance make it clear that although there is a large effect of study stimulus form (i.e., picture vs word), there is virtually no effect of test stimulus form nor of instruction. Thus, whether subjects must indicate they have seen pictures before by saying "yes" to the identical picture or "yes" to its name is irrelevant to their recognition performance; similarly, whether they must say "no" to the name of a new picture or "no" to the new picture itself is also irrelevant. Finally, whether or not they know in advance the criterion for reporting oldness or newness is also irrelevant.

\section{DISCUSSION}

The main findings of the present research are as follows: (1) Changing the criteria by which old and new concepts are classified as old or new does not change memory for pictures and words, as long as test form is the defining criterion for identifying false alarms. (2) Instructing subjects prior to study about the classification criteria used does not affect performance either when criteria are those normally used in recognition memory (control conditions) or when criteria are opposite from normal (experimental conditions). (3) The only conditions in which form of test stimulus has a significant effect are for correct rejections of old concepts (PP and WW trials for experimental groups and WP and PW trials for control groups). In these cases, old concepts tested as pictures have higher correct rejection rates than old concepts tested as words.

The first result, that the memory advantage enjoyed by pictures is unaffected by test modality, suggests that pictures are superior to words in producing better conceptual rather than sensory memory. The experimental groups showed identical performance to control groups in both old item and new item recognition. Experimental subjects were able to recognize old picture concepts tested as words equally well as control subjects recognized old picture concepts tested as pictures. Furthermore, experimental subjects were able to recognize new concepts presented as words equally well as control subjects recognized new concepts presented as pictures. It is as if the changed criterion had the effect of transforming all words to pictures and all pictures to words for the experimental groups.

The second finding, that instructions had no effect on recognition performance, is harder to interpret. Our original hypothesis was that instructed subjects would encode stimuli differently from noninstructed subjects in that they would be more likely to store both the concept itself and the form class in which it was presented, since test items would have to be distinguished according to both the original form class and the concept itself. In fact, however, the performance of instructed subjects did not differ from that of uninstructed subjects. The lack of an instructional effect suggests that form class is encoded relatively automatically along with the concept itself.

The third finding, that both groups of subjects showed higher correct rejection rates of previously presented items tested as pictures, is the only shred of evidence that form of test has any effect at all on recognition memory. Indeed, this finding is the only suggestion that the sensory code for pictures helps recognition memory performance, since no evidence of sensory code facilitation was observed for hits and false alarms.

The fact that only a small amount of sensory code facilitation was observed here, in contrast to the results of Nelson et al. (1976), may be due to the fact that the present stimuli were exceedingly simple line drawings. The drawings used by Nelson's group were considerably more complex than the present set, and this difference in the two sets of drawings may account for the discrepancy.

In summary, the present results suggest that the pictorial superiority effect in recognition memory is attributable primarily to the superiority of pictures in establishing conceptual memory codes, rather than to their superiority in being distinguished at the sensory code level as either old or new items.

\section{REFERENCES}

Jenkins, J. R., Neale, D. C., \& Deno, S. L. Differential memory for picture and word stimuli. Joumal of Educational Psychology, 1967, 58, 303-307.

KuĆERA, H., \& Francis, W. N. Computational analysis of present-day American English. Providence, R.I: Brown University Press, 1967.

Nelson, D. L., Reed, V., \& W Wlling, J. R. The pictorial superiority effect. Journal of Experimental Psychology: Human Learning and Memory, 1976, 2. 523-528.

Paivio, A. Mental imagery in associative learning and memory. Psychological Revien, 1969, 76, 241-263.

PaIvio, A. Imagery in recall and recognition. In J. Brown (Ed.), Recall and recognition. New York: Wiley, in press.

Snodgrass, J. G., \& McClure, P. Storage and retrieval properties of dual codes for pictures and words in recognition memory. Journal of Experimental Psychology: Human Learning and Memory. 1975. 1. 521-529.

(Received for publication February 4, 1977.) 